\title{
A GAMMA FUNCTION IN TWO VARIABLES
}

\author{
MOHAMED EL BACHRAOUI
}

\begin{abstract}
We introduce a gamma function $\Gamma(x, z)$ in two complex variables which extends the classical gamma function $\Gamma(z)$ in the sense that $\lim _{x \rightarrow 1} \Gamma(x, z)=$ $\Gamma(z)$. We will show that many properties which $\Gamma(z)$ enjoys extend in a natural way to the function $\Gamma(x, z)$. Among other things we shall provide functional equations, a multiplication formula, and analogues of the Stirling formula with asymptotic estimates as consequences.
\end{abstract}

\section{INTRODUCTION}

Throughout, let $\mathbb{N}, \mathbb{Z}, \mathbb{R}$, and $\mathbb{C}$ be the sets of positive integers, integers, real numbers, and complex numbers respectively. Further, let $\mathbb{N}_{0}=\mathbb{N} \cup\{0\}, \mathbb{Z}_{0}^{-}=\mathbb{Z} \backslash \mathbb{N}$, $\mathbb{R}^{+}=\mathbb{R} \backslash\{r \in \mathbb{R}: r \leq 0\}$, and $\mathcal{D}=\mathbb{C} \backslash\{x \in \mathbb{R}: x \leq 0\}$. The gamma function $\Gamma(z)$ is one of the most important special functions in mathematics with applications in many disciplines like Physics and Statistics. It was first introduced by Euler in the integral form

$$
\Gamma(z)=\int_{0}^{\infty} t^{z-1} e^{-t} d t
$$

Well-known equivalent definitions for the gamma function include the following three forms:

$$
\begin{gathered}
\Gamma(z)=\left(z e^{z \gamma} \prod_{n=1}^{\infty}\left(1+\frac{z}{n}\right) e^{-\frac{z}{n}}\right)^{-1}, \\
\Gamma(z)=\lim _{n \rightarrow \infty} \frac{n^{z} n !}{(z)_{n+1}}, \\
\Gamma(z)=\frac{1}{z} \prod_{n=1}^{\infty}\left(1+\frac{1}{n}\right)^{z}\left(1+\frac{z}{n}\right)^{-1},
\end{gathered}
$$

where $\gamma$ is the Euler-Mascheroni constant

$$
\gamma=\lim _{n \rightarrow \infty}\left(1+\frac{1}{2}+\ldots+\frac{1}{n}-\log n\right)
$$

and $(z)_{n}$ is the Pochhammer symbol

$$
(z)_{n}= \begin{cases}1 & \text { if } n=0 \\ z(z+1) \ldots(z+n-1) & \text { if } n \in \mathbb{N} .\end{cases}
$$

Date: September 11, 2021.

1991 Mathematics Subject Classification. 33B15, 11Y60, $11 \mathrm{M} 06$.

Key words and phrases. Gamma function, generalized gamma function, functional equations, special functions. 
The gamma function satisfies the basic functional equation $\Gamma(z+1)=z \Gamma(z)$. Barnes [2] and Post 11] investigated the theory of difference equations of the more general form $\phi(z+1)=f(z) \phi(z)$ under conditions on the function $f(z)$ and obtained generalized gamma functions as solutions. See also Barnes [2] where multiple gamma functions have been introduced. Many mathematicians considered concrete cases of generalized gamma functions. Dilcher [7] introduced for any nonnegative integer $k$ the function

$$
\Gamma_{k}(z):=\lim _{n \rightarrow \infty} \frac{\exp \left\{\frac{\log ^{k+1} n}{k+1} z\right\} \prod_{j=1}^{n} \exp \left\{\frac{1}{k+1} \log ^{k+1} j\right\}}{\prod_{j=0}^{n} \exp \left\{\frac{1}{k+1} \log ^{k+1}(j+z)\right\}}
$$

which for $k=0$ becomes $\Gamma(z)$, see formula (3). Diáz and Pariguan [4] extended the integral representation (10) to the function

$$
\Gamma_{k}(z)=\int_{0}^{\infty} t^{z-1} e^{-\frac{t^{k}}{k}} d t \quad\left(k \in \mathbb{R}^{+}\right)
$$

which for $k=1$ is nothing else but $\Gamma(z)$. Recently Loc and Tai 9 ] involved polynomials to define

$$
\Gamma_{f}(z)=\int_{0}^{\infty} f(t)^{z-1} e^{-t} d t
$$

which for $f(t)=t$ clearly gives $\Gamma(z)$.

In this paper we present a gamma function $\Gamma(x, z)$ in two complex variables which is meromorphic in both variables and which satisfies $\lim _{x \rightarrow 1} \Gamma(x, z)=\Gamma(z)$. Our motivation is to extend the Weierstrass form (2) in much the same way the Hurwitz zeta function

$$
\zeta(x, s)=\sum_{n=0}^{\infty} \frac{1}{(n+x)^{s}}
$$

extends the Riemann zeta function

$$
\zeta(s)=\sum_{n=1}^{\infty} \frac{1}{n^{s}} .
$$

So our definition involves the infinite product

$$
\prod_{n=0}^{\infty}\left(1+\frac{z}{n+x}\right)^{-1} e^{\frac{z}{n+x}} \quad \text { rather than } \prod_{n=1}^{\infty}\left(1+\frac{z}{n}\right)^{-1} e^{\frac{z}{n}}
$$

and in order to maintain valid the analogues of properties of $\Gamma(z)$ the factor $e^{-z \gamma}$ will be replaced by $e^{-z \gamma(x)}$, where $\gamma(x)$ is defined as follows.

Definition 1. For $x \in \mathcal{D} \backslash \mathbb{Z}_{0}^{-}$let the function $\gamma(x)$ be

$\gamma(x)=\lim _{n \rightarrow \infty}\left(\frac{1}{x}+\frac{1}{x+1}+\ldots+\frac{1}{x+n-1}-\log n\right)=\frac{1}{x}+\sum_{n=1}^{\infty}\left(\frac{1}{x+n}-\log \frac{n+1}{n}\right)$.

Note that $\gamma(1)=\gamma$ and that $\gamma(x)=\gamma_{0}(x)=-\psi(x)$ where

$$
\gamma_{0}(x)=\lim _{n \rightarrow \infty}\left(\frac{1}{x}+\frac{1}{x+1}+\ldots+\frac{1}{x+n}-\log (n+x)\right)
$$

is the zeroth Stieltjes constant and

$$
\psi(x)=\log ^{\prime} \Gamma(x)=\frac{\Gamma^{\prime}(x)}{\Gamma(x)}
$$


is the digamma function. For an account of these functions we refer to Coffey [3] and Dilcher [6]. It is easily seen that the function $\gamma(x)$ represents an analytic function on $\mathbb{C} \backslash \mathbb{Z}_{0}^{-}$and that

$$
\gamma(x+1)=\frac{-1}{x}+\gamma(x) .
$$

In section 2 we study the function $G(x, z)$ represented as an infinite product. This prepares the ground for section 3 where we introduce the gamma function $\Gamma(x, z)$ along with some of its basic properties including functional equations and a formula for the modulus $|\Gamma(n+i, n+i)|$ for $n \in \mathbb{N}_{0}$. Section 4 is devoted to the analogues of the forms (3) and (4) together with their consequences such as values at halfintegers and residues at poles. In section 5 we give the analogue of the Gauss' duplicate formula. Further in section 6 we present the analogue of the Stirling's formula leading to asymptotic estimates for our function. Finally in section 7 we give series expansions in both variables and as a result we provide recursive formulas for the coefficients of the series in terms of the Riemann-Hurwitz zeta functions.

\section{The FUnCtion $G(x, z)$}

Definition 2. For $x \in \mathbb{C} \backslash \mathbb{Z}_{0}^{-}$and $z \in \mathbb{C}$ let the function $G(x, z)$ be defined as follows

$$
G(x, z)=\prod_{\mathrm{n}=0}^{\infty}\left(1+\frac{z}{n+x}\right) e^{-\frac{z}{n+x}} .
$$

Note that $G(x, z)$ is entire in $z$ for fixed $x \in \mathbb{C} \backslash \mathbb{Z}_{0}^{-}$and that $\lim _{z \rightarrow 0} G(x, z)=$ $G(x, 0)=1$.

Proposition 1. We have:

$$
\begin{aligned}
& \text { (a) } G(x, z-1)=(z+x-1) e^{\gamma(x)} G(x, z) . \\
& \text { (b) } G(x-1, z)=\frac{z+x-1}{x-1} e^{-\frac{z}{x-1}} G(x, z) .
\end{aligned}
$$

Proof. (a) Clearly the zeros of $G(x, z)$ are $-x,-(x+1),-(x+2), \ldots$ and the zeros of $G(x, z-1)$ are $-(x-1),-x,-(x+1),-(x+2), \ldots$ Then by the theory of Weierstrass products, we can write

$$
G(x, z-1)=e^{g(x, z)}(z+x-1) \prod_{n=0}^{\infty}\left(1+\frac{z}{x+n}\right) e^{-\frac{z}{x+n}}
$$

for an entire function $g(x, z)$. Taking logarithms and differentiating with respect to $z$ we find

$$
\frac{d}{d z} \log G(x, z-1)=\frac{d}{d z} g(x, z)+\frac{1}{z+x-1}+\sum_{\mathrm{n}=0}^{\infty}\left(\frac{1}{z+x+n}-\frac{1}{x+n}\right) .
$$

On the other hand, from the definition of $G(x, z)$ we have

$$
\begin{gathered}
\frac{d}{d z} \log G(x, z-1)=\sum_{\mathrm{n}=0}^{\infty}\left(\frac{1}{z+x+n-1}-\frac{1}{x+n}\right) \\
=\frac{1}{z+x-1}-\frac{1}{x}+\sum_{\mathrm{n}=0}^{\infty}\left(\frac{1}{z+x+n}-\frac{1}{x+n}\right)+\sum_{\mathrm{n}=0}^{\infty}\left(\frac{1}{x+n}-\frac{1}{x+n+1}\right),
\end{gathered}
$$


which gives

$$
\frac{d}{d z} \log G(x, z-1)=\frac{1}{z+x-1}+\sum_{\mathrm{n}=0}^{\infty}\left(\frac{1}{z+x+n}-\frac{1}{x+n}\right) .
$$

Then the relations (6) and (7) imply that $\frac{d}{d z} g(x, z)=0$ and so $g(x, z)$ is independent of $z$, say $g(x, z)=g(x)$. It remains to prove that $g(x)=\gamma(x)$. From $G(x, z-1)=$ $(z+x-1) e^{g(x)} G(x, z)$ and $G(x, 0)=1$ we get

$$
e^{-g(x)}=x G(x, 1)=x \prod_{\mathrm{n}=0}^{\infty}\left(\frac{x+n+1}{x+n}\right) e^{-\frac{1}{x+n}} .
$$

Furthermore,

$$
\begin{aligned}
x \prod_{m=0}^{n-1}\left(\frac{x+m+1}{x+m}\right) e^{-\frac{1}{x+m}} & =(x+n) e^{-\left(\frac{1}{x}+\frac{1}{x+1}+\ldots+\frac{1}{x+n-1}\right)} \\
& =x e^{-\left(\frac{1}{x}+\frac{1}{x+1}+\ldots+\frac{1}{x+n-1}\right)}+n e^{-\left(\frac{1}{x}+\frac{1}{x+1}+\ldots+\frac{1}{x+n-1}\right)},
\end{aligned}
$$

which yields

$$
e^{-g(x)}=\lim _{n \rightarrow \infty} x \prod_{m=0}^{n-1}\left(\frac{x+m+1}{x+m}\right) e^{-\frac{1}{x+m}}=\lim _{n \rightarrow \infty} n e^{-\left(\frac{1}{x}+\frac{1}{x+1}+\ldots+\frac{1}{x+n-1}\right)},
$$

or equivalently,

$$
g(x)=\lim _{n \rightarrow \infty}\left(\frac{1}{x}+\frac{1}{x+1}+\ldots+\frac{1}{x+n-1}-\log n\right)=\gamma(x),
$$

as desired.

Part (b) follows directly by the definition of $G(x, z)$. This completes the proof.

Proposition 2. If $x \in \mathbb{C} \backslash \mathbb{Z}$, then

$$
G(x,-z) G(-x, z)=\frac{(z-x) \sin \pi(z-x)}{x \sin \pi x} e^{z \cot (\pi x)+\frac{z}{x}} .
$$

Proof. As the zeros of $\sin (z-x)$ are $x, \pi+x,-\pi+x, 2 \pi+x,-2 \pi+x, \ldots$, by the theory of Weierstrass products we have

(8) $\sin (z-x)=(z-x) e^{g(x, z)} \prod_{n=1}^{\infty}\left(1-\frac{z}{n \pi+x}\right) e^{\frac{z}{n \pi+x}} \prod_{n=1}^{\infty}\left(1+\frac{z}{n \pi-x}\right) e^{-\frac{z}{n \pi-x}}$

for an entire function $g(x, z)$. We find $e^{g(x, z)}$ as follows. Setting

$$
f_{n}(x, z)=e^{g(x, z)}(z-x) \prod_{k=1}^{n}\left(1-\frac{z}{k \pi+x}\right) e^{\frac{z}{k \pi+x}}\left(1+\frac{z}{k \pi-x}\right) e^{-\frac{z}{k \pi+x}},
$$

we have $\sin (z-x)=\lim _{n \rightarrow \infty} f_{n}(x, z)$. Taking logarithms and differentiating we obtain

$$
\begin{aligned}
\frac{f_{n}^{\prime}(x, z)}{f_{n}(x, z)} & =\frac{d}{d z} g(x, z)+\frac{1}{z-x}+\sum_{k=1}^{n}\left(\frac{-1}{k \pi+x-z}+\frac{1}{k \pi-x+z}+\frac{1}{k \pi+x}-\frac{1}{k \pi-x}\right) \\
& =\frac{d}{d z} g(x, z)+\frac{1}{z-x}+\sum_{k=1}^{n} \frac{2(x-z)}{(k \pi)^{2}-(x-z)^{2}}-\sum_{k=1}^{n} \frac{2 x}{(k \pi)^{2}-x^{2}} .
\end{aligned}
$$


But as is well-known,

$$
\lim _{n \rightarrow \infty} \frac{f_{n}^{\prime}(x, z)}{f_{n}(x, z)}=\cot (z-x)=\frac{1}{z-x}+\sum_{n=1}^{\infty} \frac{2(z-x)}{(z-x)^{2}-(n \pi)^{2}} .
$$

Thus

$$
\frac{d}{d z} g(x, z)=\sum_{n=1}^{\infty} \frac{2 x}{(k \pi)^{2}-x^{2}}=\frac{1}{x}-\cot x,
$$

and hence $g(x, z)=z\left(\frac{1}{x}-\cot x\right)+h(x)$. Now equation (8) gives

$$
\frac{\sin (z-x)}{z-x}=e^{h(x)} e^{\frac{z}{x}-z \cot x} \prod_{n=1}^{\infty}\left(1-\frac{z}{n \pi+x}\right) e^{\frac{z}{n \pi+x}}\left(1+\frac{z}{n \pi-x}\right) e^{-\frac{z}{n \pi-x}}
$$

which by letting $z \rightarrow 0$ implies

$$
e^{h(x)}=\frac{\sin x}{x}
$$

Therefore we have

$$
\frac{\sin (z-x)}{z-x}=\frac{\sin x}{x} e^{\frac{z}{x}-z \cot x} \prod_{n=1}^{\infty}\left(1-\frac{z}{n \pi+x}\right) e^{\frac{z}{n \pi+x}}\left(1+\frac{z}{n \pi-x}\right) e^{\frac{-z}{n \pi-x}} .
$$

In particular,

$$
\begin{aligned}
\frac{\sin \pi(z-x)}{\pi(z-x)} & =\frac{\sin \pi x}{\pi x} e^{\frac{z}{x}-\pi z \cot \pi x} \prod_{n=1}^{\infty}\left(1-\frac{z}{n+x}\right) e^{\frac{z}{n+x}} \prod_{n=1}^{\infty}\left(1+\frac{z}{n-x}\right) e^{\frac{-z}{n-x}} \\
& =\frac{\sin \pi x}{\pi x} e^{\frac{z}{x}-\pi z \cot \pi x} G(x,-z) G(-x, z)\left(1-\frac{z}{x}\right)^{-2} e^{-\frac{2 z}{x}} \\
& =\frac{\sin \pi x}{\pi x} e^{\frac{z}{x}-\pi z \cot \pi x} \frac{x^{2}}{(x-z)^{2}} G(x,-z) G(-x, z),
\end{aligned}
$$

or equivalently

$$
G(x,-z) G(-x, z)=\frac{(z-x) \sin \pi(z-x)}{x \sin \pi x} e^{z \cot (\pi x)+\frac{z}{x}} .
$$

This completes the proof.

Corollary 1. If $x \in \mathbb{C} \backslash \mathbb{Z}$, then

$$
\prod_{n=1}^{\infty}\left(1-\frac{z^{2}}{(n+x)^{2}}\right)\left(1-\frac{z^{2}}{(n-x)^{2}}\right)=\left(\frac{x}{\sin \pi x}\right)^{2} \frac{\sin ^{2} \pi z-\sin ^{2} \pi x}{z^{2}-x^{2}} .
$$

Proof. By the first identity in (9) we have

$$
\frac{\sin \pi(z-x)}{\pi(z-x)} \frac{\sin \pi(z+x)}{\pi(z+x)}=\left(\frac{\sin \pi x}{\pi x}\right)^{2} \prod_{n=1}^{\infty}\left(1-\frac{z^{2}}{(n+x)^{2}}\right)\left(1-\frac{z^{2}}{(n-x)^{2}}\right),
$$

which means that

$$
\prod_{n=1}^{\infty}\left(1-\frac{z^{2}}{(n+x)^{2}}\right)\left(1-\frac{z^{2}}{(n-x)^{2}}\right)=\frac{x^{2}}{z^{2}-x^{2}} \frac{\sin ^{2} \pi z-\sin ^{2} \pi x}{\sin ^{2} \pi x},
$$

which completes the proof. 


\section{The FUnCtion $\Gamma(x, z)$}

Throughout for any $x \in \mathbb{C}$ let

$$
S_{x}=\mathbb{C} \backslash\left\{-x+n: n \in \mathbb{N}_{0} \cup\{-1\}\right\} .
$$

Definition 3. For $x \in \mathbb{C} \backslash \mathbb{Z}_{0}^{-}$and $z \in S_{x}$ let the function $\Gamma(x, z)$ be defined as follows.

$$
\Gamma(x, z)=\left((z+x-1) e^{z \gamma(x)} G(x, z)\right)^{-1} .
$$

Note that for fixed $x \in \mathbb{C} \backslash \mathbb{Z}_{0}^{-}$the function $\Gamma(x, z)$ is meromorphic with simple poles at $z \in S_{x}$ and that $\lim _{x \rightarrow 1} \Gamma(x, z)=\Gamma(1, z)=\Gamma(z)$.

Proposition 3. We have
(a) $\Gamma(x, z+1)=(z+x-1) \Gamma(x, z), \quad\left(x \in \mathbb{C} \backslash \mathbb{Z}_{0}^{-}, z+1 \in S_{x}\right)$
(b) $\Gamma(x+1, z)=\frac{z+x-1}{x} \Gamma(x, z), \quad\left(x+1 \in \mathbb{C} \backslash \mathbb{Z}_{0}^{-}, z \in S_{x}\right)$
(c) $\Gamma(x+1, z+1)=\frac{(z+x-1)(z+x)}{x} \Gamma(x, z), \quad\left(x+1 \in \mathbb{C} \backslash \mathbb{Z}_{0}^{-}, z+1 \in S_{x+1}\right)$.

Proof. (a) We have

$$
\begin{aligned}
\Gamma(x, z+1) & =\left((z+x) e^{(z+1) \gamma(x)} G(x, z+1)\right)^{-1} \\
& =\left((z+x) e^{\gamma(x)} G(x, z+1) e^{z \gamma(x)}\right)^{-1} \\
& =\left(G(x, z) e^{z \gamma(x)}\right)^{-1} \\
& =(z+x-1) \Gamma(x, z),
\end{aligned}
$$

where the fourth identity follows by Proposition 1(a).

(b) We have

$$
\begin{aligned}
\Gamma(x+1, z) & =\left((z+x) e^{z \gamma(x+1)} G(x+1, z)\right)^{-1} \\
& =\left((z+x) e^{z\left(\frac{-1}{x}+\gamma(x)\right)} G(x+1, z)\right)^{-1} \\
& =\left((z+x) e^{-\frac{z}{x}} G(x+1, z) e^{z \gamma(x)}\right)^{-1} \\
& =\frac{1}{x}\left(e^{z \gamma(x)} G(x, z)\right)^{-1} \\
& =\frac{z+x-1}{x} \Gamma(x, z),
\end{aligned}
$$

where the second identity follows from the relation ( 5 ) and the fourth identity from Proposition 1(b).

(c) This part follows by a combination of part (a) and part (b). 
Corollary 2. Let $x \in \mathbb{C} \backslash \mathbb{Z}_{0}^{-}$and let $n \in \mathbb{N}$. Then we have

$$
\begin{aligned}
& \text { (a) } \quad \Gamma(x, 1)=1 \\
& \text { (b) } \quad \Gamma(x, 0)=\frac{1}{x-1}, \quad(x \neq 1) \\
& \text { (c) } \quad \Gamma(x, n)=(x)_{n-1}, \quad(n \geq 2) \\
& \text { (d) } \quad \Gamma(x,-n)=\frac{1}{(x-n-1)_{n+1}}, \\
& \text { (e) } \quad \Gamma(n, z)=\frac{(z)_{n-1}}{(n-1) !} \Gamma(z), \quad(n \geq 2) .
\end{aligned}
$$

Proof. (a) As $G(x, 0)=1$, we have by Proposition 1(a)

$$
1=x e^{\gamma(x)} G(x, 1),
$$

and thus by definition

$$
\Gamma(x, 1)=\left(x e^{\gamma(x)} G(x, 1)\right)^{-1}=1
$$

Parts (b) and (c) follow directly from Proposition 3(a). As to part (d) combine part (b) and Proposition 3(a). As to part (e) combine Proposition 3 (b) with the fact that $\Gamma(1, z)=\Gamma(z)$.

Proposition 4. We have

$$
\begin{gathered}
\text { (a) } \Gamma(x, 1-z) \Gamma(1-x, z)=\frac{-\sin \pi x}{(z-x) \sin \pi(z-x)} . \\
\text { (b) } \Gamma(x, z) \Gamma(-x,-z)=\frac{-x \sin \pi x}{\left((z+x)^{3}-(z+x)\right) \sin \pi(z+x)} .
\end{gathered}
$$

Proof. (a) We have

$$
\begin{aligned}
\Gamma(x, 1-z) \Gamma(1-x, z) & =\left((-z+x) e^{(1-z) \gamma(x)}(z-x) e^{z \gamma(1-x)} G(x, 1-z) G(1-x, z)\right)^{-1} \\
& =\frac{-e^{-\gamma(x)} e^{z \gamma(x)} e^{-z\left(\frac{1}{x}+\gamma(-x)\right)}}{(z-x)^{2}}\left(\frac{e^{-\gamma(x)}}{-z+x} \frac{-x e^{\frac{-z}{x}}}{z-x} G(x,-z) G(-x, z)\right)^{-1} \\
& =\frac{-e^{z \gamma(x)} e^{-z\left(\frac{1}{x}+\gamma(-x)\right)}}{x e^{-\frac{z}{x}(z-x) \sin \pi(z-x)} e^{z \cot \pi x-\frac{z}{x}}} \\
& =-\frac{e^{z(\gamma(x)-\gamma(-x))}}{e^{z \cot \pi x-\frac{z}{x}}} \frac{\sin \pi x}{(z-x) \sin \pi(z-x)}
\end{aligned}
$$

By Corollary 2(a, b), the previous relation gives for $z=1$

$$
\frac{1}{x-1}=\frac{-e^{\gamma(x)-\gamma(-x)-\cot \pi x+\frac{1}{x}}}{1-x} \frac{\sin \pi x}{\sin \pi(1-x)}=\frac{-e^{\gamma(x)-\gamma(-x)-\cot \pi x+\frac{1}{x}}}{1-x}
$$

which implies that

$$
e^{\gamma(x)-\gamma(-x)-\cot \pi x+\frac{1}{x}}=1
$$

giving part (a).

(b) By Proposition 3(a, b) we have

$$
\Gamma(1-x, z)=\frac{z-x-1}{-x} \Gamma(-x, z) \text { and } \Gamma(x, 1-z)=(-z+x-1) \Gamma(x,-z) .
$$


Then by virtue of part (a) we get

$$
\frac{z-x-1}{-x} \Gamma(-x, z)(-z+x-1) \Gamma(x,-z)=\frac{-\sin \pi x}{(z-x) \sin \pi(z-x)},
$$

or equivalently

$$
\Gamma(-x, z) \Gamma(x,-z)=\frac{-x \sin \pi x}{\left((z-x)^{3}-(z-x)\right) \sin \pi(z-x)} .
$$

This completes the proof.

Corollary 3. If $n \in \mathbb{N}_{0}$ and $z \notin \mathbb{N}_{0}$, then

$$
\lim _{x \rightarrow-n} \Gamma(x, z)=0 .
$$

Proof. By Proposition 4(a) we have

$$
\Gamma(x, z)=\Gamma(x, 1-(1-z))=\frac{-\sin \pi x}{(1-z-x) \sin \pi(1-z-x)} \frac{1}{\Gamma(1-x, 1-z)} .
$$

Then

$$
\lim _{x \rightarrow-n} \Gamma(x, z)=\frac{-\sin \pi n}{(1-z+n) \sin \pi(1-z+n)} \frac{1}{\Gamma(1+n, 1-z)}=0 .
$$

Corollary 4. If $n \in \mathbb{N}_{0}$, then

$$
|\Gamma(n+i, n+i)|^{2}=|\Gamma(n-i, n-i)|^{2}=\frac{5 \prod_{k=0}^{2 n-2}\left(4+k^{2}\right)}{\prod_{k=0}^{n-1}\left(1+k^{2}\right)} \frac{e^{\pi}}{10\left(e^{2 \pi}+1\right)} .
$$

Proof. First note that

$$
\Gamma(\bar{x}, \bar{z})=\overline{\Gamma(x, z)},
$$

from which the first identity immediately follows. As to the second formula, using identity (10) and Proposition 4(b) we obtain

$$
|\Gamma(i, i)|^{2}=\Gamma(i, i) \overline{\Gamma(i, i)}=\Gamma(i, i) \Gamma(\bar{i}, \bar{i})=\frac{-i \sin \pi i}{\left((2 i)^{3}-(2 i)\right) \sin 2 \pi i}=\frac{e^{\pi}}{10\left(e^{2 \pi}+1\right)},
$$

which gives the result for $n=0$. If $n>1$ we have by Proposition 3(c)

$$
\begin{gathered}
|\Gamma(n+i, n+i)|^{2}=\Gamma(n+i, n+i) \Gamma(n-i, n-i) \\
=\frac{(2 i-1)(2 i) \ldots(2 i+2 n-2)}{i(i+1) \ldots(i+n-1)} \frac{(-2 i-1)(-2 i) \ldots(-2 i+2 n-2)}{-i(-i+1) \ldots(-i+n-1)}|\Gamma(i, i)|^{2} \\
=\frac{(-1)^{2 n}(2 i-1)(2 i+1)(2 i)(2 i)(2 i+1)(2 i-1) \ldots(2 i+2 n-2)(2 i-(2 n-2))}{(-1)^{n}(i)(i)(i+1)(i-1) \ldots(i+n-1)(i-(n-1))}|\Gamma(i, i)|^{2} \\
=\frac{(4+1)(4)(4+1) \ldots\left(4+(2 n-2)^{2}\right)}{1(1+1) \ldots\left(1+(n-1)^{2}\right)} \frac{e^{\pi}}{10\left(e^{2 \pi}+1\right)} .
\end{gathered}
$$

This completes the proof. 


\section{Analogues of Euler's formulas, Residues, And VAlues at} HALF-INTEGERS

Proposition 5. We have

(a) $\Gamma(x, z)=\lim _{n \rightarrow \infty} \frac{n^{z} x(x+1) \ldots(x+n-1)}{(z+x-1)(z+x) \ldots(z+x+n-1)}=\lim _{n \rightarrow \infty} \frac{n^{z}(x)_{n}}{(z+x-1)_{n+1}}$.

$$
\text { (b) } \Gamma(x, z)=\frac{x}{(z+x-1)(z+x)} \prod_{n=1}^{\infty}\left(1+\frac{1}{n}\right)^{z}\left(1+\frac{z}{x+n}\right)^{-1} \text {. }
$$

Proof. (a) We have

$$
\begin{aligned}
\Gamma(x, z) & =\lim _{n \rightarrow \infty}\left((z+x-1) e^{z\left(\frac{1}{x}+\frac{1}{x+1}+\ldots+\frac{1}{x+n-1}-\log n\right)} \prod_{k=0}^{n-1}\left(1+\frac{z}{x+k}\right) e^{-\frac{z}{x+k}}\right)^{-1} \\
& =\lim _{n \rightarrow \infty}\left((z+x-1) e^{-z \log n} \prod_{k=0}^{n-1} \frac{z+x+k}{x+k}\right)^{-1} \\
& =\lim _{n \rightarrow \infty}\left(n^{-z} \frac{(z+x-1)(z+x) \ldots(z+x+n-1)}{x(x+1) \ldots(x+n-1)}\right)^{-1} \\
& =\lim _{n \rightarrow \infty} \frac{n^{z} x(x+1) \ldots(x+n-1)}{(z+x-1)(z+x) \ldots(z+x+n-1)}
\end{aligned}
$$

(b) By the previous proof we have

$$
\begin{aligned}
\Gamma(x, z) & =\frac{1}{z+x-1} \lim _{n \rightarrow \infty} n^{z} \prod_{k=0}^{n-1}\left(1+\frac{z}{x+k}\right)^{-1} \\
& =\frac{1}{z+x-1} \lim _{n \rightarrow \infty} \prod_{k=1}^{n-1}\left(1+\frac{1}{k}\right)^{z} \prod_{k=0}^{n-1}\left(1+\frac{z}{x+k}\right)^{-1} \\
& =\frac{x}{(z+x-1)(z+x)} \prod_{n=1}^{\infty}\left(1+\frac{1}{n}\right)^{z}\left(1+\frac{z}{x+n}\right)^{-1} .
\end{aligned}
$$

Corollary 5. If $x, x+z \in \mathbb{C} \backslash \mathbb{Z}_{0}^{-}$, then

$$
\Gamma(x, z) \Gamma(x+z,-z)=\frac{1}{(x-1)(z+x-1)} .
$$

Proof. By Proposition 5(a) we have

$\Gamma(x, z)=\lim _{n \rightarrow \infty} \frac{n^{z}(x)_{n}}{(z+x-1)_{n+1}}=\frac{1}{x-1} \lim _{n \rightarrow \infty} \frac{n^{z}(x-1)_{n+1}}{(z+x-1)_{n+1}}=\frac{1}{z+x-1} \lim _{n \rightarrow \infty} \frac{n^{z}(x)_{n+1}}{(z+x)_{n+1}}$,

where the last identity follows since $\lim _{n \rightarrow \infty} \frac{x+n}{z+x+n}=1$. Then

$$
\Gamma(x+z,-z)=\frac{1}{x-1} \lim _{n \rightarrow \infty} \frac{n^{-z}(x+z)_{n+1}}{(x)_{n+1}}=\frac{1}{(x-1)(z+x-1) \Gamma(x, z)} .
$$

This completes the proof. 
Corollary 6. If $k, l \in \mathbb{N}_{0}$ such that $k+l \neq 0$, then

$$
\Gamma\left(\frac{2 k+1}{2}, \frac{2 l+1}{2}\right)=\frac{2}{\sqrt{\pi}(2 k-1)} \frac{(2 l+2) !}{(-4)^{l+1}(l+1) !} \frac{(k+l-1) !}{\left(-l-\frac{1}{2}\right)_{k+l}}
$$

Proof. On the one hand we have by Corollary 5

$$
\Gamma\left(\frac{2 k+1}{2}, \frac{2 l+1}{2}\right) \Gamma\left(k+l+1,-\frac{2 l+1}{2}\right)=\frac{2}{(2 k-1)(k+l)} .
$$

On the other hand by Corollary 2(e) and the well-known fact that

$$
\Gamma(1 / 2-k)=\frac{\sqrt{\pi}(-4)^{k} k !}{(2 k) !}
$$

we have

$$
\begin{gathered}
\Gamma\left(k+l+1,-\frac{2 l+1}{2}\right)=\frac{(-l-1 / 2)_{k+l}}{(k+l) !} \Gamma(-l-1 / 2) \\
=\frac{(-l-1 / 2)_{k+l}}{(k+l) !} \Gamma(1 / 2-(l+1))=\frac{(-l-1 / 2)_{k+l}}{(k+l) !} \frac{\sqrt{\pi}(-4)^{l+1}(l+1) !}{(2 l+2) !} .
\end{gathered}
$$

Now combine these identities to deduce the required formula.

Corollary 7. If $x \in \mathbb{C} \backslash \mathbb{Z}$ and $m \in \mathbb{N}_{0} \cup\{-1\}$, then the residue of $\Gamma(x, z)$ at $z=-(x+m)$ is

$$
\begin{cases}\frac{1}{(x-1) \Gamma(x-1)}, & \text { if } m=-1 \\ \frac{(-1)^{m+1}(x)_{2 m+1}}{(m+1) !} \frac{1}{\Gamma(x+2 m+1)}, & \text { otherwise. }\end{cases}
$$

Proof. Suppose first that $m=-1$. By Corollary 5 and Proposition 3(c) we obtain

$$
\Gamma(x, z)=\frac{1}{(x-1)(z+x-1)} \frac{1}{\Gamma(z+x,-z)} .
$$

Then

$$
\lim _{z \rightarrow-(x-1)}(z+(x-1)) \Gamma(x, z)=\lim _{z \rightarrow-(x-1)} \frac{1}{(x-1) \Gamma(1, x-1)}=\frac{1}{(x-1) \Gamma(x-1)} .
$$

Suppose now that $m \neq-1$. Then repeatedly application of Proposition 3(c) yields

$$
\begin{gathered}
\Gamma(x, z)=\frac{1}{(x-1)(z+x-1)} \frac{1}{\Gamma(z+x,-z)} \\
=\frac{x}{(z+x-1)(z+x)} \frac{1}{\Gamma(z+x+1,-z+1)} \\
=\frac{(x)_{2 m+1}}{(z+x-1)_{m+2}} \frac{1}{\Gamma(z+x+m+1,-z+m+1)},
\end{gathered}
$$

or equivalently,

$$
(z+x+m) \Gamma(x, z)=\frac{(x)_{2 m+1}}{(z+x-1)_{m+1}} \frac{1}{\Gamma(z+x+m+1,-z+m+1)} .
$$

Thus

$$
\lim _{z \rightarrow-(x+m)}(z+x+m) \Gamma(x, z)=\frac{(x)_{2 m+1}}{(-1)^{m+1}(m+1) !} \frac{1}{\Gamma(1, x+2 m+1)},
$$

which implies the desired result since $\Gamma(1, x+2 m+1)=\Gamma(x+2 m+1)$. 
Note that if $x=1$ and $m=-1,0,1,2, \ldots$, then Corollary 7 agrees with the well-known fact that the residue of $\Gamma(z)$ at $z=-(m+1)$ is

$$
\frac{(-1)^{m+1}}{(m+1) !}
$$

5. An analogues of the Gauss' multiplication formula

Proposition 6. If $x \in \mathbb{C} \backslash \mathbb{Z}_{0}^{-}$, then the function

$$
f(x, z)=\frac{n^{n z} \Gamma(x, z) \Gamma\left(x, z+\frac{1}{n}\right) \ldots \Gamma\left(x, z+\frac{n-1}{n}\right)}{n \Gamma(n(x-1)+1, n z)}
$$

is independent of $z$.

Proof. By Proposition 5 we have

$$
\begin{gathered}
f(x, z)=\frac{n^{n z} \prod_{k=0}^{n-1} \lim _{m \rightarrow \infty} \frac{m^{z+\frac{k}{n}}(x)_{m-1}}{\left(z+\frac{k}{n}+x-1\right)\left(z+\frac{k}{n}+x\right) \ldots\left(z+\frac{k}{n}+x+m-2\right)}}{n \lim _{m \rightarrow \infty} \frac{(m n)^{n z}(n(x-1))_{m n-1}}{(n z+n(x-1))_{m n}}} \\
=\lim _{m \rightarrow \infty} \frac{n^{m n-1} m^{\frac{n-1}{2}}\left((x)_{m-1}\right)^{n-1}}{(n(x-1))_{m n-1}} \frac{(n(z+x-1))_{m n}}{\prod_{k=0}^{n-1} \prod_{j=0}^{m-1}(n(z+x-1)+k+j n)} \\
=\lim _{m \rightarrow \infty} \frac{n^{m n-1} m^{\frac{n-1}{2}}\left((x)_{m-1}\right)^{n-1}}{(n(x-1))_{m n-1}}
\end{gathered}
$$

where the last identity follows as

$$
\frac{(n(z+x-1))_{m n}}{\prod_{k=0}^{n-1} \prod_{j=0}^{m-1}(n(z+x-1)+k+j n)}=1 .
$$

This completes the proof.

Corollary 8. We have

$$
\begin{gathered}
\Gamma(x, z) \Gamma\left(x, z+\frac{1}{2}\right) \Gamma(1-x, z) \Gamma\left(1-x, z+\frac{1}{2}\right) \\
=2^{2-4 z} \Gamma(2 x-1,2 z) \Gamma(1-2 x, 2 z) \frac{\tan \pi x}{x-\frac{1}{2}} .
\end{gathered}
$$

Proof. Taking $z=\frac{1}{n}$ in Proposition 6 we obtain

$$
\begin{gathered}
f(x, z) f(1-x, z)=f\left(x, \frac{1}{n}\right) f\left(1-x, \frac{1}{n}\right) \\
=\frac{\Gamma\left(x, \frac{1}{n}\right) \Gamma\left(x, \frac{2}{n}\right) \ldots \Gamma\left(x, \frac{n-1}{n}\right)}{\Gamma(n(x-1)+1,1)} \frac{\Gamma\left(1-x, \frac{1}{n}\right) \Gamma\left(1-x, \frac{2}{n}\right) \ldots \Gamma\left(1-x, \frac{n-1}{n}\right)}{\Gamma(-n x+1,1)} \\
=\Gamma\left(x, \frac{1}{n}\right) \Gamma\left(1-x, \frac{n-1}{n}\right) \Gamma\left(x, \frac{2}{n}\right) \Gamma\left(1-x, \frac{n-1}{n}\right) \ldots \Gamma\left(x, \frac{n-1}{n}\right) \Gamma\left(1-x, \frac{1}{n}\right) \\
=\frac{(-1)^{n-1} \sin \pi x}{\prod_{k=1}^{n-1}\left(\left(\frac{k}{n}-x\right) \sin \pi\left(\frac{k}{n}-x\right)\right)},
\end{gathered}
$$


where the last identity follows by Proposition 4(a). Now if $n=2$, then Proposition 6 combined with the previous formula gives

$$
2^{4 z-2} \frac{\Gamma(x, z) \Gamma\left(x, z+\frac{1}{2}\right) \Gamma(1-x, z) \Gamma\left(1-x, z+\frac{1}{2}\right)}{\Gamma(2 x-1,2 z) \Gamma(1-2 x, 2 z)}=\frac{-\sin \pi x}{\left(\frac{1}{2}-x\right) \sin \left(\frac{\pi}{2}-\pi x\right)},
$$

or equivalently,

$\Gamma(x, z) \Gamma\left(x, z+\frac{1}{2}\right) \Gamma(1-x, z) \Gamma\left(1-x, z+\frac{1}{2}\right)=2^{2-4 z} \Gamma(2 x-1,2 z) \Gamma(1-2 x, 2 z) \frac{\tan \pi x}{x-\frac{1}{2}}$.

This completes the proof.

\section{An analogue of the Stirling's formula}

In this section we essentially use the same ideas as in Lang [8, p. 422-427] to derive a formula for $\log \Gamma(x, z)$ leading to asymptotic formulas for $\Gamma(x, z)$ which are analogues to the Stirling's formula. For $t \in \mathbb{R}$, let $P(t)=t-\lfloor t\rfloor-\frac{1}{2}$ and for convenience for $z \in \mathcal{D}$ let

$$
I_{n}(z)=\int_{0}^{n} \frac{P(t)}{z+t} d t, \quad \text { and } \quad I(z)=\lim _{n \rightarrow \infty} I_{n}(z)=\int_{0}^{\infty} \frac{P(t)}{z+t} d t .
$$

Proposition 7. If $x \in \mathbb{R}^{+}$and $z \in \mathbb{R}^{+} \cap S_{x}$, then

$\log \Gamma(x, z)=\left(z+x-\frac{3}{2}\right) \log (z+x-1)-z+1-\left(x-\frac{1}{2}\right) \log x+I(x)-I(z+x-1)$.

Proof. We have with the help of Euler's summation formula

$$
\begin{aligned}
& \log \frac{(z+x-1)(z+x) \ldots(z+x+n-1)}{x(x+1) \ldots(x+n)}=\sum_{k=0}^{n} \log (z+x-1+k)-\sum_{k=0}^{n} \log (x+k) \\
& =\int_{0}^{n} \log (z+x-1+t) d t+\frac{1}{2}(\log (z+x-1+n)+\log (z+x-1))+I_{n}(z+x-1) \\
& \quad-\int_{0}^{n} \log (x+t) d t-\frac{1}{2}(\log (x+n)+\log x)-I_{n}(x) \\
& =[(z+x-1+t) \log (z+x-1+t)-(z+x-1+t)]_{0}^{n}-[(x+t) \log (z+x-1+t)-(x+t)]_{0}^{n} \\
& +\frac{1}{2}(\log (z+x-1+n)+\log (z+x-1))-\frac{1}{2}(\log (x+n)+\log (x))+I_{n}(z+x-1)-I_{n}(x),
\end{aligned}
$$

which after routine calculations becomes

$$
\begin{gathered}
\log \frac{(z+x-1)(z+x) \ldots(z+x+n-1)}{x(x+1) \ldots(x+n)}=\log n^{z}+z \log \left(1+\frac{z+x-1}{n}\right) \\
+\left(x+n-\frac{3}{2}\right) \log \left(1+\frac{z+x-1}{n}\right)-\left(z+x-\frac{3}{2}\right) \log (z+x-1)-\left(x+n+\frac{1}{2}\right) \log \left(1+\frac{x}{n}\right) \\
+\left(x-\frac{1}{2}\right) \log x-\log n+I_{n}(z+x-1)-I_{n}(x) .
\end{gathered}
$$

Equivalently,

$$
\begin{gathered}
\log \frac{(z+x-1)(z+x) \ldots(z+x+n-1)}{n^{z} x(x+1) \ldots(x+n-1)}=\log (x+n)+z \log \left(1+\frac{z+x-1}{n}\right) \\
+\left(x+n-\frac{3}{2}\right) \log \left(1+\frac{z+x-1}{n}\right)-\left(z+x-\frac{3}{2}\right) \log (z+x-1)-\left(x+n+\frac{1}{2}\right) \log \left(1+\frac{x}{n}\right)
\end{gathered}
$$




$$
\begin{gathered}
+\left(x-\frac{1}{2}\right) \log x-\log n+I_{n}(z+x-1)-I_{n}(x) \\
=z \log \left(1+\frac{z+x-1}{n}\right)+\left(x+n-\frac{3}{2}\right) \log \left(1+\frac{z+x-1}{n}\right)-\left(z+x-\frac{3}{2}\right) \log (z+x-1) \\
-\left(x+n-\frac{1}{2}\right) \log \left(1+\frac{x}{n}\right)+\left(x-\frac{1}{2}\right) \log x+I_{n}(z+x-1)-I_{n}(x) .
\end{gathered}
$$

Now use the fact that

$$
\log \left(1+\frac{z}{n}\right)=\frac{z}{n}+O\left(\frac{1}{n^{2}}\right)
$$

and Proposition 5(a) and take $\lim _{n \rightarrow \infty}$ on both sides to get

$\log \frac{1}{\Gamma(x, z)}=(z+x-1)-\left(z+x-\frac{3}{2}\right) \log (z+x-1)-x+\left(x-\frac{1}{2}\right) \log x+I(z+x-1)-I(x)$,

implying the required identity.

Corollary 9. Let $x \in \mathbb{R}^{+}$and $z \in \mathbb{R}^{+} \cap S_{x}$. Then

(a) for $x \rightarrow \infty$ we have

$$
\Gamma(x, z) \sim(z+x-1)^{z+x-3 / 2} e^{1-z} x^{1 / 2-x},
$$

(b) for $z \rightarrow \infty$ we have

$$
\Gamma(x, z) \sim(z+x-1)^{z+x-3 / 2} e^{1-z} x^{1 / 2-x}+I(x) .
$$

Proof. Combine Proposition 7 with the fact that $\lim _{z \rightarrow \infty} I(z)=0$.

\section{SERIES EXPANSIONS AND RECURSIVE FORMULAS FOR THE COEFFICIENTS}

To use the property $\log \left(z_{1} z_{2}\right)=\log z_{1}+\log z_{2}$, we suppose in this section that $x \in \mathbb{R}^{+}$and $z \in \mathbb{R}^{+} \cap S_{x}$.

Proposition 8. If $|z|<\inf (1,|x|)$, then

$$
\log \Gamma(x, z+1)=-z \gamma(x)-\sum_{m=2}^{\infty} \frac{(-1)^{m-1}}{m} \zeta(m, x) z^{m} .
$$

Proof. On the one hand, we have by definition

$$
\log \Gamma(x, z)=-\log (z+x-1)-z \gamma(x)-\sum_{\mathrm{n}=0}^{\infty}\left(\log \left(1+\frac{z}{x+n}\right)-\frac{z}{x+n}\right) .
$$

On the other hand, by Proposition 3(a) we have

$$
\log \Gamma(x, z+1)=-\log (z+x-1)+\log \Gamma(x, z) .
$$


Combining these two relations we obtain

$$
\begin{aligned}
\log \Gamma(x, z+1) & =-z \gamma(x)-\sum_{\mathrm{n}=0}^{\infty}\left(\log \left(1+\frac{z}{x+n}\right)-\frac{z}{x+n}\right) \\
& =-z \gamma(x)-\sum_{\mathrm{n}=0}^{\infty}\left(\left(\sum_{m=1}^{\infty} \frac{-(1)^{m-1}}{m} \frac{z^{m}}{(x+n)^{m}}\right)-\frac{z}{x+n}\right) \\
& =-z \gamma(x)-\sum_{m=2}^{\infty} \frac{(-1)^{m-1}}{m} z^{m} \sum_{\mathrm{n}=0}^{\infty} \frac{1}{(x+n)^{m}} \\
& =-z \gamma(x)-\sum_{m=2}^{\infty} \frac{(-1)^{m-1}}{m} \zeta(m, x) z^{m} .
\end{aligned}
$$

Corollary 10. If $|z|<\inf (1,|x|)$ and

$$
\Gamma(x, z+1)=\sum_{m=0}^{\infty} a_{m}(x) z^{m},
$$

then $a_{0}(x)=1$ and for $m>0$ we have

$$
a_{m}(x)=\frac{1}{m}\left(-a_{m-1}(x) \gamma(x)+\sum_{k=0}^{m-2}(-1)^{m} a_{k}(x) \zeta(m-k, x)\right) .
$$

Proof. Clearly if $z=0$, then $\Gamma(x, 1)=a_{0}(x)=1$ by Corollary 2(a). Differentiating the power series with respect to $z$ gives

$$
\frac{d}{d z} \Gamma(x, z+1)=\sum_{m=1}^{\infty} m a_{m}(x) z^{m-1}
$$

Further in Proposition 8 differentiating with respect to $z$ yields

$$
\frac{d}{d z} \log \Gamma(x, z+1)=\frac{\frac{d}{d z} \Gamma(x, z+1)}{\Gamma(x, z+1)}=-\gamma(x)-\sum_{m=2}^{\infty}(-1)^{m-1} \zeta(m, x) z^{m-1} .
$$

Next combining (11) with (12) gives

$$
\sum_{m=1}^{\infty} m a_{m}(x) z^{m-1}=\left(\sum_{m=0}^{\infty} a_{m}(x) z^{m}\right)\left(-\gamma(x)+\sum_{m=2}^{\infty}(-1)^{m} \zeta(m, x) z^{m-1}\right) .
$$

Now the desired identity follows by equating the coefficients.

Proposition 9. If $|x-1|<\inf (1,|z+1|)$, then

$$
\begin{gathered}
\log \Gamma(x+1, z)=\sum_{n=1}^{\infty}\left(z \log \frac{n+1}{n}-\log \frac{z+n}{n}\right) \\
+\sum_{n=2}^{\infty} \frac{z(x-1)}{n(n+z)}+\sum_{m=2}^{\infty} \frac{(-1)^{m}\left(\zeta(m, z)-\zeta(m)-\frac{1}{z^{m}}+1\right)}{m}(x-1)^{m} .
\end{gathered}
$$


Proof. Note that it is easily checked that

$$
\begin{gathered}
-z \gamma(x)+\sum_{n=2}^{\infty}\left(\frac{z}{x+n-1}-\frac{n+z}{n}\right) \\
=-\frac{z}{x}+\log (1+z)+\sum_{n=1}^{\infty}\left(z \log \frac{n+1}{n}-\log \frac{n+z}{n}\right) .
\end{gathered}
$$

Now combining the definition of $\Gamma(x, z)$ with Proposition 3(b) yields

$$
\begin{aligned}
& \log \Gamma(x+1, z)=-\log x-z \gamma(x) \\
& -\sum_{\mathrm{n}=0}^{\infty}\left(\log (x+n+z)-\log (x+n)-\frac{z}{x+n}\right) \\
& =-z \gamma(x)-\log (x+z)+\frac{z}{x} \\
& -\sum_{n=2}^{\infty}\left(\log (x-1+n+z)-\log (x-1+n)-\frac{z}{x-1+n}\right) \\
& =-z \gamma(x)-\log (x+z)+\frac{z}{x} \\
& -\sum_{n=2}^{\infty}\left(\log (n+z)+\log \left(1+\frac{x-1}{n+z}\right)-\log n-\log \left(1+\frac{x-1}{n}\right)-\frac{z}{x-1+n}\right) \\
& =-z \gamma(x)+\sum_{n=2}^{\infty}\left(\frac{z}{x-1+n}-\log \frac{n+z}{n}\right)-\log (x+z)+\frac{z}{x} \\
& -\sum_{n=2}^{\infty}\left(\log \left(1+\frac{x-1}{n+z}\right)-\log \left(1+\frac{x-1}{n}\right)\right) \\
& =\log (1+z)-\log (x-1+z+1)+\sum_{n=1}^{\infty}\left(z \log \frac{n+1}{n}-\log \frac{n+z}{n}\right) \\
& +\sum_{n=2}^{\infty} \sum_{m=1}^{\infty} \frac{(-1)^{m-1}}{m}\left(\frac{1}{n^{m}}-\frac{1}{(n+z)^{m}}\right)(x-1)^{m} \\
& =-\log \left(1+\frac{x-1}{z+1}\right)+\sum_{n=1}^{\infty}\left(z \log \frac{n+1}{n}-\log \frac{n+z}{n}\right)+\sum_{n=2}^{\infty} \frac{z(x-1)}{n(n+z)} \\
& +\sum_{m=2}^{\infty} \frac{(-1)^{m-1}}{m}\left(-1+\zeta(m)+\frac{1}{z^{m}}+\frac{1}{(z+1)^{m}}-\zeta(m, z)\right)(x-1)^{m} \\
& =\sum_{n=1}^{\infty}\left(z \log \frac{n+1}{n}-\log \frac{n+z}{n}\right) \\
& +\sum_{n=2}^{\infty} \frac{z(x-1)}{n(n+z)}+\sum_{m=2}^{\infty} \frac{(-1)^{m-1}}{m}\left(-1+\zeta(m)+\frac{1}{z^{m}}-\zeta(m, z)\right)(x-1)^{m},
\end{aligned}
$$

where the fifth identity follows with the help of (13). This completes the proof. 
Corollary 11. If $|x-1|<\inf (1,|z+1|)$ and

$$
\Gamma(x+1, z)=\sum_{m=0}^{\infty} b_{m}(z)(x-1)^{m},
$$

then $b_{0}(z)=z \Gamma(z)$ and for $m>0$

$$
\begin{gathered}
b_{m}(z)=\frac{1}{m} b_{m-1}(z)+\sum_{n=2}^{\infty} \frac{z}{n(n+z)} \\
+\frac{1}{m} \sum_{k=0}^{m-2}(-1)^{m-k} b_{k}(z)\left(\zeta(m-k, z)-\zeta(m-k)-z^{-(m-k)}+1\right) .
\end{gathered}
$$

Proof. Taking $x=1$, we have $b_{0}(z)=\Gamma(2, z)=z \Gamma(z)$ by Corollary $2(\mathrm{e})$. Further, by Proposition 9 we have

$$
\begin{gathered}
\frac{d}{d x} \log \Gamma(x+1, z)=\frac{\frac{d}{d x} \Gamma(x+1, z)}{\Gamma(x+1, z)} \\
=\sum_{n=2}^{\infty} \frac{z}{n(n+z)}+\sum_{m=2}^{\infty}(-1)^{m}\left(\zeta(m, z)-\zeta(m)-z^{-m}+1\right)(x-1)^{m-1} .
\end{gathered}
$$

On the other hand, it follows from the assumption that

$$
\frac{d}{d x} \Gamma(x+1, z)=\sum_{m=1}^{\infty} m b_{m}(z)(x-1)^{m-1} .
$$

Then from (14) and (15) we get

$$
\begin{gathered}
\sum_{m=1}^{\infty} m b_{m}(z)(x-1)^{m-1}=\left(\sum_{m=0}^{\infty} b_{m}(x-1)^{m}\right) \times \\
\left(\sum_{n=2}^{\infty} \frac{z}{n(n+z)}+\sum_{m=2}^{\infty}(-1)^{m}\left(\zeta(m, z)-\zeta(m)-z^{-m}+1\right)(x-1)^{m-1}\right)
\end{gathered}
$$

and the result follows by equating the coefficients.

\section{REFERENCES}

[1] E.W. Barnes, On the theory of the multiple gamma functions, Trans. Cambridge. Phil. Soc. 19 (1904), 374-425.

[2] E.W. Barnes, The Linear Difference Equation of the First Order, Proc. London Math. Soc. 2 (2) (1905), 438-469.

[3] Mark W. Coffey, Integral and series representations of the digamma and polygamma functions, Analysis 32 (4) (2012), 317-337.

[4] R. Díaz and E. Pariguan, On hypergeometric functions and Pochhammer k-symbol Divulgaciones Matemáticas 15 (2) (2007), 179-192.

[5] R. Díaz and C. Teruel, q,k-Generalized Gamma and Beta Functions, Journal of Nonlinear Mathematical Physics 12 (1) (2005), 118-134.

[6] K. Dilcher, Generalized Euler constants for arithmetical progressions, Math. Comp. 59 (1992), $259-282$.

[7] K. Dilcher, On generalized gamma functions related to the Laurent coefficients of the Riemann zeta function, Aequationes Mathematicae 48 (1994), 55-85

[8] S. Lang, Complex Analysis, Fourth Edition, Springer, 1998.

[9] T. G. Loc and T. D. Tai, The generalized gamma functions, (2011), arXiv:1105.6002

[10] J. E. Marsden and M. J. Hoffman, Basic complex analysis, Third edition, Freeman, 1998.

[11] E.L. Post, The Generalized gamma functions, Ann. Math. 20 (2) (1919), 202-217. 
[12] E. T. Whittaker and G. N. Watson, Course of modern analysis, Fourth edition, Cambridge University Press, 1996.

Dept. Math. Sci, United Arab Emirates University, PO Box 17551, Al-Ain, UAE

E-mail address: melbachraoui@uaeu.ac.ae 